\title{
KM3NeT: $\mathrm{A} \mathrm{km}^{3}$-scale neutrino telescope in the Mediterranean Sea
}

\author{
Véronique Van Elewyck*, for the KM3NeT Consortium ${ }^{\dagger}$ \\ APC (Université Paris-Diderot, CNRS/IN2P3, CEA/IRFU and Observatoire de Paris) \\ 10 rue A. Domon et L. Duquet, 75205 Paris Cedex 13, France \\ E-mail: elewyckeapc.univ-paris7.fr
}

The KM3NeT consortium aims at the construction of a research infrastructure in the Mediterranean deep sea, hosting a neutrino telescope with an instrumented volume of several $\mathrm{km}^{3}$ and an integrated platform for earth and deep sea sciences.

The telescope location in the Mediterranean Sea will allow for surveying a large part of the Galactic Plane, including the Galactic Centre, thus complementing the sky coverage of the IceCube telescope located at the South Pole. A telescope deployed in deep sea water is expected to have a very good angular resolution (of the order of $0.1^{\circ}$ for neutrinos with $100 \mathrm{TeV}$ energy), providing high sensitivity to point-like sources. The realization of this project will provide the scientific community with a powerful instrument to study and constrain the mechanisms at play in a broad range of astrophysical objects, including supernova remnants, active galactic nuclei, gamma-ray bursts and possibly also dark matter.

This challenging project will require the installation of thousands of photon detectors with their related electronics and calibration systems several kilometers below sea level. This contribution presents an outline of the technological aspects of the project and a discussion of its main physics goals and expected performances, with a proposed preliminary timeline towards the completion of this instrument.

25th Texas Symposium on Relativistic Astrophysics - TEXAS 2010

December 06-10, 2010

Heidelberg, Germany

*Speaker.

${ }^{\dagger}$ http://www.km3net.org 


\section{Introduction and motivations}

It has long been recognized that the neutrino is an interesting cosmic messenger to perform astronomy: it emerges from very dense media and travels across cosmological distances without being either deflected by the ambient magnetic fields, or scattered/absorbed by the ambient matter and radiation. High-energy $(\sim \mathrm{TeV}-\mathrm{PeV})$ cosmic neutrinos are thought to be produced in a wide range of astrophysical objects, through the interactions of accelerated protons and nuclei with matter and radiation in the vicinity of the source [1]. Their detection would therefore trace unambiguously the existence of hadronic processes inside these sources. Opening the window of neutrino astronomy could also lead to the discovery of new types of sources, yet unobserved with photons or cosmic rays.

High-energy neutrino telescopes are designed to detect the charged leptons produced when a neutrino interacts with material around the detector. The Cherenkov light emitted by these leptons when they travel in a transparent medium such as water or ice can be detected by a 3-dimensional array of photosensors. Muons are the preferred detection channel, but showers induced by electron and tau-neutrino can also be detected. The timing, position and amplitude of the hits recorded by the photosensors allow the reconstruction of the muon trajectory, providing the arrival direction of the parent neutrino and an estimation of its energy. Such detectors are installed at great depths and optimized to detect up-going muons produced by neutrinos which have traversed the Earth, in order to limit the background from down-going atmospheric muons.

Three neutrino telescopes are currently operating worldwide; the most advanced one is IceCube [2], which has recently achieved its final configuration with 86 strings, instrumenting one $\mathrm{km}^{3}$ of South pole ice. Results from the 40-string configuration (IC40) have already been issued and data from IC59 are currently under analysis; no evidence for a cosmic neutrino signal has been reported so far. Another neutrino telescope has been operating for some years in Lake Baikal [3] in a much smaller configuration; it has recently deployed 2 prototype strings for a $\mathrm{km}^{3}$-scale detector. Finally, ANTARES [4] is a neutrino telescope deployed in the Mediterranean Sea near Toulon (France); it is operating in its complete, 12-string configuration since 2008. Recent results from IceCube and ANTARES can be found elsewhere in these proceedings [5].

Two more projects have also been developing prototype instrumented units for a neutrino telescope in the sea: the NEMO collaboration has performed site characterization and deployment tests near Capo Passero in Sicily (Italy) [6], and the NESTOR collaboration off the shore of Pylos (Greece) [7]. ANTARES, NEMO and NESTOR are now part of the KM3NeT consortium which coordinates the efforts towards the building of the next-generation neutrino telescope in the Mediterranean Sea. Section 2 of this contribution briefly discusses the performance requirements imposed by the physics case of KM3NeT, as well as the technical constraints related to the operation of such a large-scale, deep-sea facility. The present status of the technical design of the detector is presented in Section 3, while Section 4 describes its expected performance and sketches the timeline of the development of the project in the upcoming years.

\section{The KM3NeT project: physics goals and technical constraints}

ANTARES, the largest neutrino telescope currently operating in the Northern Hemisphere, has an instrumented volume roughly 50 times smaller than that of IceCube, providing very limited 


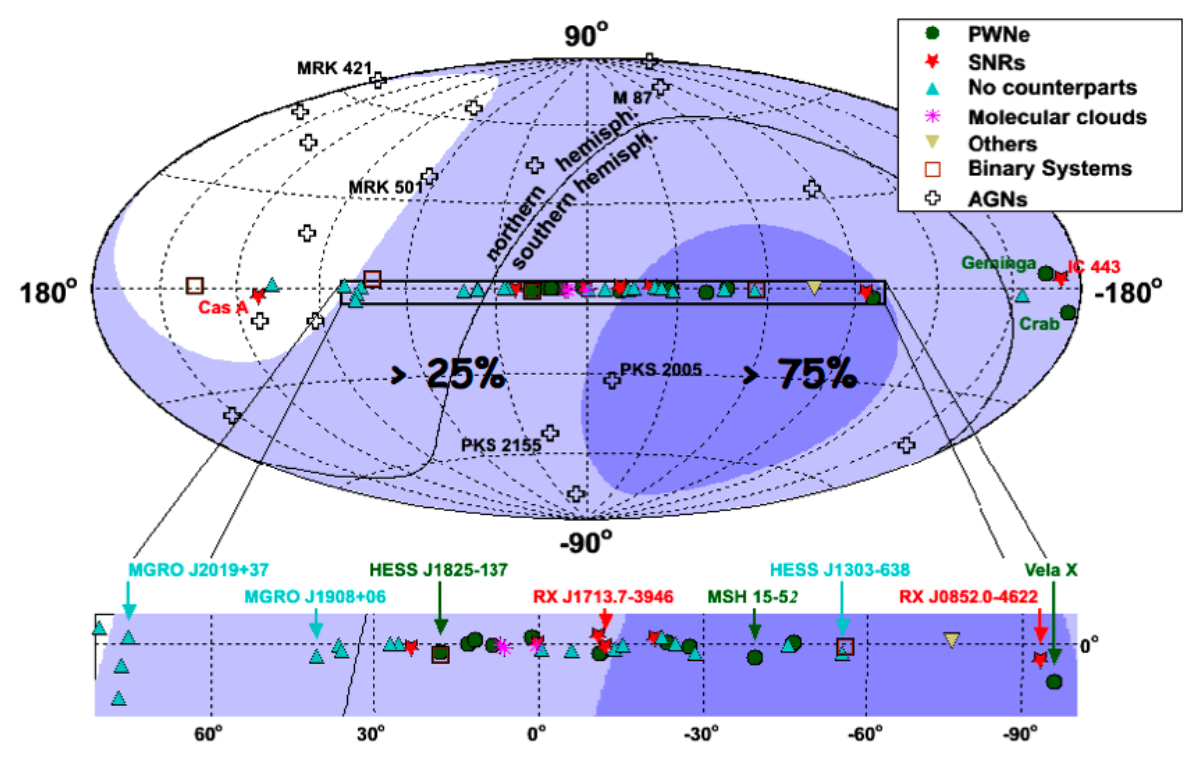

Figure 1: Integrated sky coverage in Galactic coordinates for a neutrino telescope located in the Mediterranean Sea. The dark (light) grey areas are visible at least $75 \%$ (25\%) of the time, assuming $2 \pi$ downward coverage. The locations of observed sources of high-energy $\gamma$-rays are also indicated.

potentialities to detect cosmic neutrinos. In view of the negative results reported so far by IceCube (in a configuration about half its total size), it has in fact become clear that detectors beyond the $\mathrm{km}^{3}$ scale are required to fully exploit the scientific potential of neutrino astronomy. A minimal operation time of 10 years also looks like a good compromise between the desired sensitivity reach and the realistic possibility of operation and maintenance of such a large-scale detector in a hostile deep-sea environment.

The main physics goal of KM3NeT is the observation of point-like sources of high-energy neutrinos, in particular in the energy range from 1 to $100 \mathrm{TeV}$. High-energy gamma-ray astronomy has indeed confirmed the existence of a wide range of galactic (shell-type Supernovae remnants, pulsar wind nebulae, microquasars,...) and extra-galactic (active galactic nuclei, gamma-ray bursts) sources with electromagnetic emissions at and beyond the $\mathrm{TeV}$ level. In the case of transient or flaring sources, time-correlation of neutrino events with other observations may help significantly reduce the physical background of atmospheric neutrinos, and multi-messenger strategies such as the ones used by ANTARES [8] will also be applied.

The field of view of a detector located at the latitude of the Mediterranean Sea nicely complements that of IceCube (which, in the considered range of energies, is mostly sensitive to neutrinos from the Northern Hemisphere). Figure 1 shows the integrated coverage map of KM3NeT together with the positions of known astrophysical sources of potential interest. The integrated visibility is about $3,5 \pi$ steradians. A large part of the Galactic Plane will thus be observed for a significant fraction of time, including e.g. the Galactic Center itself (which hosts a powerful TeV gamma-ray source), the Crab Nebula, the promising supernovae remnants Vela Junior and RX J1713.7-3946 and TeV sources HESS J1745-303 and J1626-490. 
The angular resolution is a critical parameter in the search for point-like sources and an important aspect to assess in the design and optimization of the detector. It is essentially determined by the performance of the algorithms used for track reconstruction, which in turn depends on the timing, charge and position accuracy of the optical units in the detector. Basic requirements concern the positioning accuracy of the photosensors $(<40 \mathrm{~cm})$, and the single-photon time resolution $(<2 \mathrm{~ns})$.

Other items such as the identification and measurement of a diffuse flux of ultra-high energy ( $>$ $100 \mathrm{PeV}$ ) neutrinos of cosmogenic origin (also dubbed as GZK neutrinos), the indirect search for signals of dark matter annihilation in the Sun and the Galactic Center, or the search for exotica such as magnetic monopoles, are also on the physics agenda of KM3NeT, although they do not drive the design effort. Finally, the project is also meant to foster the development of interdisciplinary research by providing a node for long-term measurements in marine and Earth science, that will share part of the global cabled submarine infrastructure with the neutrino telescope.

Besides the desired optimization of the detector according to physics performance and priorities, the main challenge in designing the KM3NeT infrastructure lies in the technical constraints imposed by the deployment, operation and maintenance of a large-scale electro-mechanical structure at a depth of several kilometers in the sea. All mechanical elements, and in particular the spheres enclosing the photomultipliers, should stand high pressures and have long-term resistance to a chemically aggressive environment. The vertical structures supporting the optical modules have to be sufficiently flexible to stand sea currents that can reach $30 \mathrm{~cm}$ per second. A technology for long-distance data transmission is also needed for the connection of the telescope with the shore station several tens of kilometres away.

Finally, an undersea telescope has to cope with an important optical background due to the presence of radioactive ${ }^{40} \mathrm{~K}$ in the sea salt and to natural bioluminescence. The effect of this background on the event selection and reconstruction can be reduced by imposing local coincidences between different photosensors, which also constrains the design and distribution of the optical modules along the DUs.

\section{Technical design and optimization}

In April 2008 the KM3NeT Consortium published the Conceptual Design Report (CDR) of the project [9], which presents an initial set of design concepts for the future telescope. Vertical structures supporting optical modules (OMs, i.e. photomultiplier tubes (PMTs) enclosed in a pressure-resistant glass sphere) constitute the basic detection units (DUs), which are anchored to the sea bed and kept vertical by submerged buoys. They are connected to shore via a network of electro-optical cables and junction boxes lying on the sea floor. The OMs are distributed along the DUs in clusters mounted on a mechanical frame (or storey), in order to improve the reconstruction efficiency and to reduce the impact of the optical backgrounds mentioned above. All OMs in a DU are connected to the same backbone cable, which serves both for power and data transport and terminates in one of the junction boxes. Due to the extreme deep-sea conditions, the connection of the DUs to the central network must be performed using a remotely operated vehicle (ROV).

Different technical solutions have since been investigated in view of the established physics priorities and their estimated cost, leading to the writing of the KM3NeT Technical Design Report 
(TDR) [10], which concluded the Design Study Phase. Some of the main issues addressed in the TDR concern:

- The optical module. The proposed approaches were a conservative single-PMT OM with a single large (8") PMT enclosed in a 13" glass sphere, and a novel "multi-PMT" solution consisting of a 17" glass sphere filled with 31 small (3") PMTs, and their front-end electronics, as shown in figure 2 (left panel). The overall photocathode area of one such multi-PMT OM exceeds that of a standard single-PMT OM by at least a factor 3 . In this scenario, all signals are digitized inside the $\mathrm{OM}$ and sent to the DU backbone cable through a single penetrator. The multi-PMT design also provides very good separation between single- and multi-photon hits, together with directional information which is expected to significantly improve the track reconstruction performance. In view of these assets, and of the results of validation tests performed on various prototypes, this option has recently been retained as the most promising one for the final design of the detector.

- The structure of the detector units. Three design options inspired from the pilot projects were investigated; these differ essentially in the horizontal extent of the 'storeys', which may consist either of a single multi-PMT OM, a circular mechanical structure with 6 OMs in a triangular configuration (a conservative solution similar to the ANTARES-triangular geometry [4]), or an extended horizontal "bar" Ĺ la NEMO [6]. Such bars are arranged perpendicularly to each other into a flexible "tower" stabilized by a trapezoidal geometry of ropes, as shown in the middle panel of fig. 2. Such a configuration has the advantage of breaking the azimuthal symmetry of the DU, allowing for the reconstruction of muon tracks whose Cherenkov cone has hit OMs in only one detection unit and thereby increasing the sensitivity of the detector at low and intermediate energies. The tower design has also been retained recently as the most appropriate for the final layout of the detector. The final length and spacing of the bars have been set respectively to $6 \mathrm{~m}$ and 40 $\mathrm{m}$ as a compromise between the increased sensitivity of the detector and the stability and manageability of the structure. A deployment test of a compactified, selfunfurling tower was successfully performed in Spring 2010. Fig. 2 (middle and right panel) shows a possible implementation of the two favoured options: a tower made of bar-like storeys with two multi-PMT OMs at their ends.
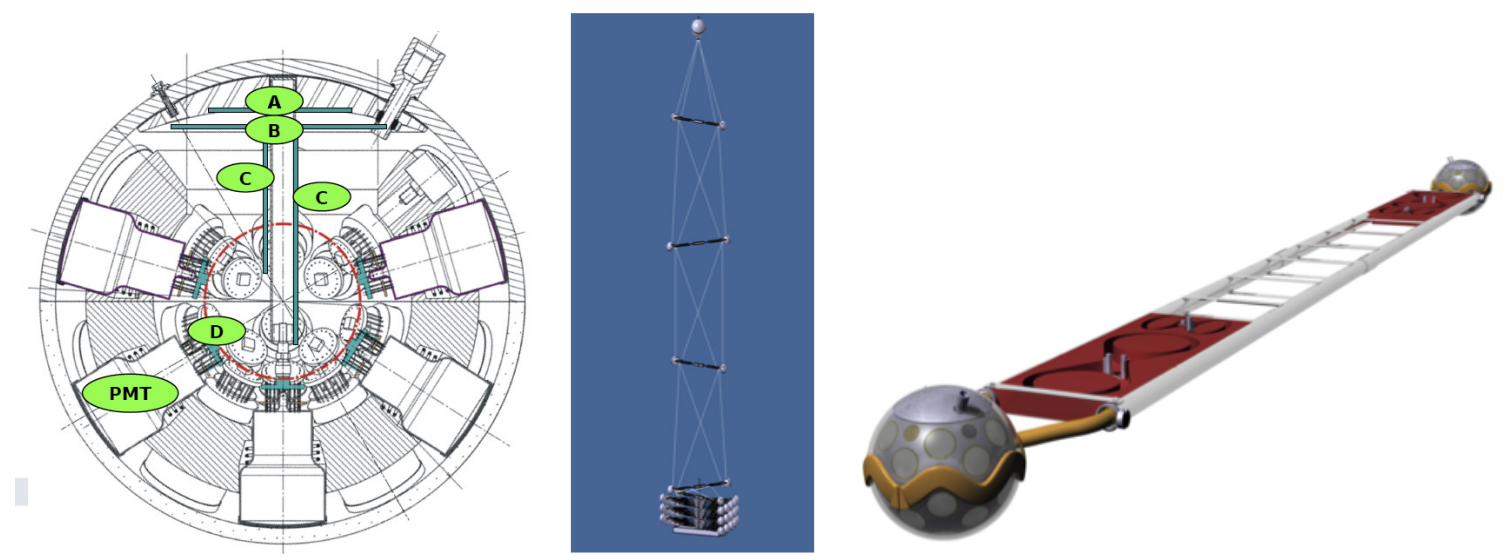

Figure 2: Left: schematic drawing of a multi-PMT OM, showing the cooling structure (A), the front-end electronic components (B,C) and the PMT bases (D). Middle: schematic view of a detector unit with a tower structure, showing the possible positions for OMs and the stabilizing ropes between adjacent orthogonal storeys. Right: possible implementation of a bar-like storey supporting two multi-PMT optical modules. 
- The data readout scheme. The central front-end component of the readout is the SCOTT chip (for Sampler of Comparators Outputs with Time Tagging), a dedicated ASIC currently under development. It digitizes the times of threshold crossing of the analogue PMT signal, converting it into time-over-threshold information. Up to 16 channels are available per chip; they can be used either to set different thresholds on the same PMT (thereby allowing to reconstruct the shape of the signal) or to treat in parallel the outputs of several PMTs from the same multi-PMT OM. An FPGA processor then collects the digitized signals and converts them to optical signals which are multiplexed using the DWDM (Dense Wavelength Division Multiplexing) technology to be sent to shore via optical fibres. Following the strategy adopted by ANTARES, no local data filtering is implemented: all digitized hits are sent to shore where they are then filtered according to different trigger algorithms.

An in-depth discussion of these and other technical aspects, including the deep-sea and shore infrastructure and the design and operation of the node for associated sciences, as well as general aspects of quality control and risk assessment, can be found in the KM3NeT Technical Design Report [10] and in other recent papers on the subject [11].

For the design options discussed above, full Monte Carlo simulations have been performed for signal neutrinos, atmospheric neutrinos and atmospheric muons, taking into account the full process chain from the expected flux at sea level through the detection of Cherenkov light to the selection and the reconstruction of events. Such simulations have been used extensively to optimize the detector design, and in particular its geometrical parameters. For the tower-like design, sensitivity vs. cost studies have shown that a regular grid of about 250 DUs spaced by $180 \mathrm{~m}$ appears to give the best performance results while fitting into the overall estimated budget of about $220 \mathrm{M} €$. Such a detector would correspond to an instrumented volume of approximatively $5 \mathrm{~km}^{3}$, thereby surpassing significantly the size of IceCube.

\section{Expected performance and timeline}

Monte Carlo simulations have also been used to estimate the angular resolution (defined as the median of the separation angle between the incoming neutrino and the reconstructed muon track) and the sensitivity of the detector. At low energies $(\leq \mathrm{TeV})$, the angular resolution is essentially determined by the intrinsic angle between the neutrino and the muon and is of the order of a degree; but it improves rapidly with energy, as a result of the good transmission properties of sea water. It reaches $0.2^{\circ}$ at a neutrino energy of about $30 \mathrm{TeV}$, and becomes better than $01^{\circ}$ above $100 \mathrm{TeV}$.

The sensitivity is usually expressed in terms of a neutrino effective area, which translates into a discovery or exclusion potential for a given hypothesis of neutrino flux. Figure 3 shows the sensitivity of the KM3NeT detector to point-like sources with a standard assumption of an unbroken power-law flux $\Phi\left(E_{v}\right)=\Phi_{0}\left(E_{v} / 1 \mathrm{TeV}\right)^{-2}$. It is to be noted, however, that the optimization of the quality cuts and of the reconstruction algorithm has a significant impact on the achievable sensitivity and is still subject to ongoing studies. In any case, KM3NeT will increase significantly the sensitivity to neutrino point sources in the Southern Hemisphere, and in particular to potential galactic sources such as those mentioned in Section 1, for which the handful of events required for a significant detection could be reached over 5 years observation time. GRBs appear as another promising target, as the requirement of a time coincidence allows a drastic reduction of the background which counterbalances the smallness of the expected signal. 


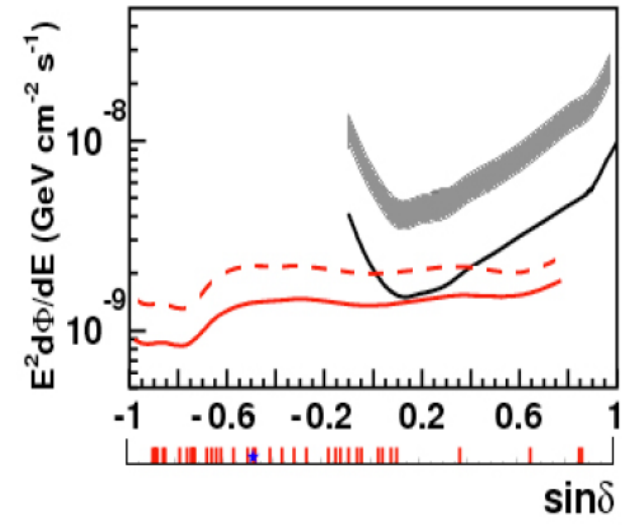

Figure 3: Sensitivity of the full KM3NeT detector to neutrino point sources with an $E^{-2}$ spectrum for one year of observation, as a function of the source declination $\delta$. The red lines indicate the flux sensitivity ( $90 \%$ C.L.; full line) and the discovery flux ( $5 \sigma, 50 \%$ probability; dashed line), as inferred from a binned analysis method. Also shown is the IceCube flux sensitivity for one year (black line) and the IceCube discovery flux $(5 \sigma, 50 \%$ probability) as extrapolated from the IC40 configuration (shaded band, spanning a factor 2.5 to 3.5 above the flux sensitivity. The red ticks at the bottom of the horizontal axis show the positions of known gamma-ray sources in the Galactic Plane; the position of the Galactic Centre is indicated by a blue star.

The upcoming year will be dedicated to further prototyping and field tests in order to converge on a final proposal by the end of the current EU-funded Preparatory Phase (in March 2012). Concurrently, simulation studies will be pursued to react to technical developments and to assess the detector footprint. The important decision on the choice of the detector site (Toulon, Capo Passero or Pylos, differing both in depth and in distance to shore), to be made by the October 2011, will also have an impact on the final design. Assuming that all funding and administrative issues are sorted out by then, production could be launched and the first DUs be deployed soon after. The fullscale detector is expected to be installed and operational by the end of 2018; but data taking will already start during the early stages of the construction, rapidly exceeding in statistics and quality all first-generation telescopes in the Mediterranean and providing an exciting discovery potential.

\section{Acknowledgements}

Many warm thanks are due to all people in charge of the workshop for the perfect organization and the wonderful venue. The author acknowledges support from the French Agence Nationale de la Recherche (contract ANR-08-JCJC-0061-01) and from the EU FP7 (Marie Curie Reintegration Grant). The KM3NeT Design Study is supported through EU FP6 (contract $n^{\circ}$ 011937) and the Preparatory Phase through EU FP7 (contract $n^{\circ} 212525$ ).

\section{References}

[1] see e.g. J. K. Becker, Phys. Rept. 458 (2008) 173 [arXiv:0710.1557 [astro-ph]] and references therein.

[2] F. Halzen and S. R. Klein, Rev. Sci. Instrum. 81 (2010) 081101 [arXiv:1007.1247 [astro-ph.HE]].

[3] A. Avrorin et al., Nucl. Instrum. Meth. A 626-627 (2011) S13.

[4] J. Brunner [ANTARES Coll.], Nucl. Instrum. Meth. A 626-627 (2011) S19.

[5] see the contributions from K. Payet (ANTARES) and A. Ishihara (IceCube), these Proceedings.

[6] M. Taiuti et al. [NEMO Coll.], Nucl. Instrum. Meth. A 626-627 (2011) S25.

[7] P. A. Rapidis [NESTOR Coll.], Nucl. Instrum. Meth. A 602 (2009) 54.

[8] V. Van Elewyck [ANTARES Coll.], Nucl. Instrum. Meth. A 626-627 (2011) S180.

[9] KM3NeT Consortium, Conceptual Design Report, ISBN 978-90-6488-031-5

[10] KM3NeT Consortium, Technical Design Report, ISBN 978-90-6488-033-9; both the CDR and the TDR are available on http://www.km3net.org .

[11] U. F. Katz [KM3NeT Coll.], Nucl. Instrum. Meth. A 626-627 (2011) S57 and references therein. 